\title{
Designing a context-aware recommender system in the optimization of the relief and rescue
}

\author{
N. Bahrami ${ }^{1, *}$, M. Argany ${ }^{2}$, N. N. Samani ${ }^{2}$, A. R. Vafaeinejad ${ }^{3}$ \\ ${ }^{1}$ Ph.D. candidate, RS \& GIS, Factually of Geography, University of Tehran, Tehran, Iran - nd.bahrami@ut.ac.ir \\ ${ }^{2}$ Department of RS \& GIS, Factually of Geography, University of Tehran, Tehran, Iran - argany@ut.ac.ir, nnsamani@ut.ac.ir \\ ${ }^{3}$ Faculty of Civil, Water and Environmental Engineering, Shahid Beheshti University, Tehran, Iran - a_vafaei@ sbu.ac.ir
}

\begin{abstract}
KEY WORDS: Context-aware, Optimization, Relief \& Rescue, Particle Swarm Optimization, Geospatial Information System, Earthquake
\end{abstract}

\begin{abstract}
:
The context-aware is the knowledge that leads to better cognition and recognition of the environment, objects and factors, and the way of communication and interactions between them. As a result, it can have a great impact in providing appropriate solutions to various problems. It is possible to integrate consciousness into relief and rescue discussions and to take steps to improve and make realistic solutions. In this study, this issue was addressed in the earthquake crisis, due to a large number of seismic faults in Iran, is one of the major crises in Iran and many parts of the world. Hence, the contexts of rescuers, teams, and environment as the main textures in the above-mentioned issue are investigated and their relationship with each other and the priorities of activities and locations by identifying specialties and the physical and situational conditions of the relief workers, and an algorithm was designed and optimized to optimize the allocation of the relief workers to the affected areas and the necessary activities. Finally, the improvement of the 2.4 fold results of the algorithm and the proposed structure of this research resulted in the ratio of non-use of this algorithm.
\end{abstract}

\section{INSTRUCTIONS}

One of the issues that every year causes many human lives around the world is natural disasters. The more unpredictable such incidents, the more so, the more dangerous they were. Among the disasters such as flood, storm, volcano, earthquake, tsunami, earthquake is an event that is more and more unpredictable than other accidents. To cope with crises and disasters, a structure called crisis management is used that addresses all the issues before, during and after the accident and leads to activities in the field of preparedness, prevention, coping (response) and reconstruction It will be. One of the most important tasks that could lead to a reduction in injuries is the disaster relief \& rescue issue, which is of the crisis management phases.

In this research, we have tried to make this necessary. In this regard, the study of the context and structure of earthquake relief and rescue, this as one of the main crises of the country due to its geographic location and its earthquake, is considered. All contexts of the rescuers, the teams, the relief workers, and the environment of the earthquake event have been investigated, and their explanations are mentioned in the following topics. The textures and information that appear during the Sense process include the location, time, amount of damage, and the interactions of the rescuers with each other and with the environment, activities, specializations, priorities, conditions and physical and situational status Rescue workers. Finally, an algorithm is used to optimize the activities and tasks allocation of the rescuers using existing information.

\section{THEORETICAL FOUNDATIONS}

2.1 Context-Aware: To develop a context-aware system for relief and rescue teams, we first need to examine the concepts of context and consciousness in this issue. The context has been studied widely. The context can be used to describe the status of an entity; an entity can be an individual, an object, or a location. Also, the context can be defined as places, identities of people or objects around the user or time-related issues (such as day, week, or season). When the concept of context is defined, three aspects are considered in the environment: object or entity, object environment and physical environment (Argany et al. 2015). The object's environment is related to behavior and its neighbors and how they relate to them. The physical environment is included calculations environment.

\subsection{Relief \& Rescue}

2.2.1 Review the tasks of the rescuers: This section examines the responsibilities of rescue workers in the earthquake crisis and important points in the earthquake relief process. Some search and rescue actors include four components of locating, evaluating, fixing, and transferring (Ezadi, 2011). First, the location and release of individuals and the medical assessment and, if necessary, the use of primary care, emergency treatment (stabilization) and transfer to treatment centers is carried out (Bahrami, 2019). The rescue team should have a precise program to carry out rescue operations for those in detention. 
2.2.2 Search and Rescue Operations Management: To ensure the success of search and rescue operations in urban areas; it must be done very carefully. The relief and rescue program can be divided into five stages, respectively (Bahrami, 2019):

- Primary Identification - Data Collection (Preliminary Assessment)

- Quickly assess the area (Technical Inspection)

- Surface Search and Rescue in the Damaged Area (Primary Rescue)

- Search and rescue by technical means (Secondary Rescue)

- The systematic removal of debris (Final Collapse Lifting)

On the other hand, seven steps in search and rescue operations are assumed to be considered by the savior's people (Valadbeygi, Pour Heydari, 2011):

1. Data collection: One of the first steps to be taken is to assess and assess the situation.

2. Evaluation of Damage: By looking at different angles to the buildings.

3. Identifying resources and accessing them: including access to facilities, equipment, and personnel.

4. Priority: Includes emergency diagnosis and safety assurance for the continuation of search and rescue operations. Sometimes a building should be marked in such a way that no other person enters it and waits for other forces or more facilities.

5. Designing a Rescue Plan: In this section, it becomes clear who and with whom the conditions will enter the building.

6. Guidance for search and rescue operations: Search for people under the rubble remains and caught

7. Evaluate progress: The situation must always be checked to assess the progress of the rescue program and to prevent any damage to the relief forces

2.3 Particle Swarm Optimization (PSO): The first attempt by Kennedy and Eberhart, after simulating the social behavior of birds in 1995, presented the particle group optimization method. The components of a group follow a simple behavior. In this way, each member of the group imitates the success of their other neighbors. The purpose of such algorithms is to move members of the group to the search space and to accumulate at an optimal point (such as the source of food) (Saeedian, 2016).

\section{LITERATURE REVIEW}

In 2019 Youngchul Shin et al., improved post-crisis transportation by integrating Ant colony algorithm and linear planning (Shin et al. 2019) and Vahidnia et al., had distributed tasks in spatial networks using a game theory (Vahidnia, 2019). In 2018, Haowei Zhang et al., introduced the entropy-based PSO algorithm for the task scheduling problem (Zhang et al. 2018). Vafaeinejad Designed a dynamic GIS for on navigation purpose in urban area (Vafaeinejad, 2018). Tang Jian et al., Have proposed a rescue solution using simulated annealing optimization (Tang et al. 2018) and also Bolouri et al., Using simulated annealing optimization to locate fire stations. (Bolouri et al. 2018); also Hongman Wang et al., Improved emergency transportation using multi-purpose ant-community algorithm (Wang et al. 2018). Mousanejad et al., Using geographic information system and simulated annealing for optimizing the railway design (Mousanejad et al., 2018) and Aghakhani et al., Using Geospatial Inforrmation System
Assessment of the effects of land use scenarios on watershed surface runoff (Aghakhani et al. 2018). Argany et al., (2018), developsd a GIS-based context-aware for optimization sensor coverage in an urban area (Argany, 2018). In 2017, Ilaria Baffo et al., Improved the Emergency Routing using an Ant Colony Algorithm (Baffo et al. 2017) and Vafaeinejad developed Spatio-temporal GIS for Dynamic Guidance of an Autonomous Vehicle (Vafaeinejad 2017). in 2016 Gyeongtaek Oh et al., has been studied to assign optimal particle swarm algorithm based tasks for scheduling collaborative activities. (Oh et al. 2016). Lei Xu et al. Have also used annealing algorithm with genetic algorithm to allocate resources for a multi-user system (Lei et al. 2016), and Akbari and Rashidi use a multi-objective scheduling algorithm based on the cuckoo algorithm in Task allocation has been used in heterogeneous systems (Akbari et al. 2016). In 2015, by Lin and Chiu used a hybrid particle swarm optimization algorithm and local search for resource allocation (Lin et al. 2015), as well as Nadia Nedjah et al., distributed PSO-based algorithm for dynamic task allocation of robots have introduced (Nedjah et al. 2015); Wei Hong et al. Have also used PSO to assign two-level tasks (Wei et al. 2015). In the same year, R.K. Jena also timed multi-tasks in the cloud with a nested PSO framework (Jena, 2015). Jaziar Radianti et al. Used a spatio-temporal modeling for fire extinguishing (Radianti et al. 2015). In 2014 the Ant colony algotithm was used by Jason Mahdjoub et al. to better coordinate rescue teams in disaster management (Mahdjoub et al. 2014). As well as Neysani Samany et al., developed a Fuzzy context-aware systems (Neysani Samanyet al., 2014). In 2013, Ole-Christoffer Granmo et al., used the DNB model to track and predict the movement of people until evacuation, and the ACO model to dynamically find safe ways to respond to secondary evacuation hazards, and dynamic spatio-temporal models have been achieved (Granmo et al. 2013) and Neysani Samanyet al., a contextaware systems for urban traffic networks using dynamic range neighbor query and directed interval algebra (Neysani Samanyet al., 2013). In 2012, Rasekh and Vafaeinejad used exponential time-lapse multi-channel queuing theory to plan earthquake rescue teams (Rasekh et al. 2012), and Abdolsalam Ghaderi et al., used a hybrid PSO to locate accommodation (Ghaderi et al. 2012) and Yao Lin Liu et al. Used PSO and multi-objective optimization techniques to allocate rural land in the semi-arid region of China (Liu et al. 2012). Xiaoping Liua et al. Used the Ant colony algorithm to optimize land use allocation in large areas (Liua et al. 2012); Vafaeinejad et al. in 2010 A New Method for Modeling and Planning Group Activities with Spatiotemporal Modeling of Activities Human groups have provided a way to increase the efficiency of collective human activities (Vafaeinejad et al. 2010). In 2009 Vafaeinejad et al. designed a spatial-temporal solution to earthquake relief and rescue (Vafaeinejad et al. 2009).

\section{METHODOLOGY}

The first step in designing and developing a context-aware application is to identify and model the Effective contexts, and how to contexts efficacy. In other words, in designed the application, one should pay attention to how the context is selected and how it affects the performance. It can increase the efficiency and usability of the program. Various sensors, such as physical and functional sensors, are the receivers of context data (Sajadian et al. 2017). Sensors in this system 
include position sensors (GPS) and timing and velocity meters to identify the individuals context and their neighbours context, and the spacing system relative to their neighbours and context damaged in the environment as well as the service Provides damage estimation. The most important issue in a context-aware system is to determine the position and continuous tracking of the user, accurately and accurately. Time is important in emergencies.

Positioning in ubiquitous systems has been discussing in many studies in recent years. In most studies has been used a multisensor system or a combination of different sensors to determine the position (Sajadian et al. 2017). In this research, the GPS has been using. The management and processing of context data are storing on the database after being sent to the processing server, and data processing is performing in this section. The processing of data in the context argumentation is based on propositions in the form of "if-then" sentences and, finally, decisions are being made by users in the recommendations based on spatial-temporal conditions (Sajadian et al. 2017).

To provide a meaningful definition for the concept of "field" in the establishment of relief and rescue teams, the concept of a rescuer has the main place in this definition. As shown in Figure 1 , the entire problem space is from a rescuer as the main body, a relief team as an object environment that includes information on a group of rescuers, including the position of the team members, their disposition from each other and the situation Physical activity and their activities are in relation to other rescuers in the group, as well as the physical environment consisting of a group of injured people, residential areas and relief and rescue teams in a particular area. There may also be links and restrictions to launch relief and rescue operations. It may also include information on the priorities for allocating aid workers and existing activities.

Therefore, a comprehensive definition of the context in the rescue and rescue theme is suggesting as follows:

"The context is a rescuer's entire status, context, or environment, including information on the rescuer, the rescue team, and the physical environment and their interactions at a given time." That is, we need to identify different context information (CI) for a focused rescue team. Knowing about CI for rescuers is an awareness of the location, speed, time, physical condition, and the relief worker's distance from activities in different locations, and their relationship with other aid workers and the physical environment.

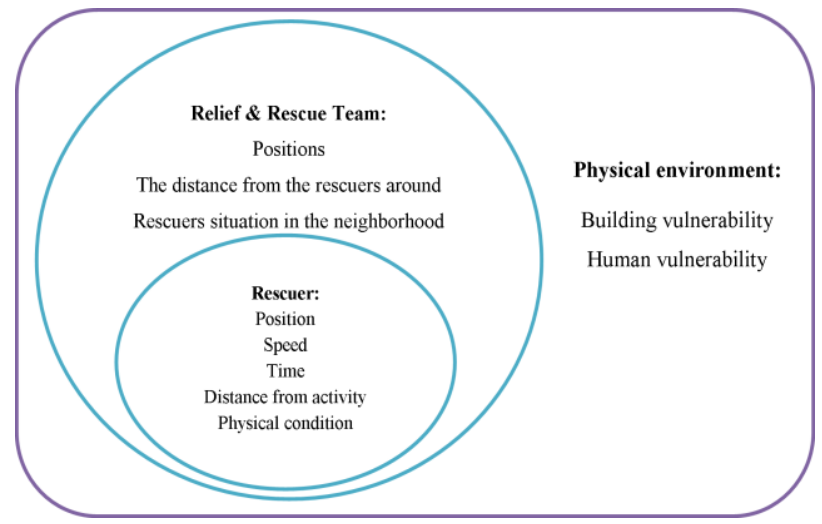

Figure 1. Three components of context in relief \& rescue

Given the framework for rescuers and rescue teams, an informed context optimization algorithm has been developed for optimization tasks allocation to improve team performance. To maximize the effectiveness of teams and reduce the relief and rescue times, in an urban environment where the earthquake has happened, the relief and rescue activities and context information recorded at any time, the main objective of the design and implementation of this algorithm. To decide on intelligence actions to optimize relief and rescue teams, different levels are assuming for CI. Considering the different $\mathrm{CI}$ and the amount of improvement obtained from the implementation of the proposed optimization algorithm, the best arrangement of rescue teams at the accident site were designed and implemented.

To achieve the relief \& rescue optimal management, close interaction is being necessary. To establish a relationship between the context information (CIs) obtained and the possibility of optimizing the relief and rescue process with this information, the mathematical relation must be defined and finally, using the Sense information from different contexts in the problem environment and the proposed algorithm, it is optimized. According to the information obtained in the research, as mentioned above, the function has designed as the objective function in this algorithm (Equation 1), which is as follows, using the CIs and the proposed algorithm for this research and the abovementioned priorities. It will be. Since this function is a continuous nonlinear function, and also according to studies, the method of optimizing the congestion of particle capabilities is solved by such functions, and the answers to this algorithm, which is the allocation of individuals to the activities in this research it is optimized:

$$
\text { Cost }=(1 / \text { MaxInjured }) *\left(\mathrm{e}^{-\mathrm{SS} \times \mathrm{ST} \times \text { Spacing } / \text { Area Assigned }}\right)
$$

In the above relationship, all parameters must follow a unit or reputation (Vafaeinejad et al. 2009); "Max Injured" the most injured number among the wounded of each residential building, "Area Assigned" is the area (Mountaineer Area Rescue Group. Probability of Detection), which in this study is the same activity of area is located. "Spacing" the relief distance to the operating area and the "ST" and "SS" are respectively the duration of the work and the speed of the relief worker. If a rescuer will sent to a region that is estimated to be several people under debris, the duration of activities will be multiplied by the number of submarines. And, the final cost of an activity that requires several people to do it will be obtained from the sum of the costs of each who performs that activity.

\section{RESULTS AND DISCUSSION}

5.1 Study Area and Dataset: The desired issue in a part of the central region of Tehran. The relief and rescue activities of the earthquake crisis include Searching, Light Collapse Lifting, Heavy Collapse Lifting, Primary Helping, Securing, Pointing, Securing Pilot, Air update in the rubble, reconstruction of the network of roads (Asgari et al. 2012, Mahdjoub et al. 2014). In this study, 32 responders were considered in the four domains of 8 people (Rasekh et al. 2012), and at the beginning of the relief, they were deployed at the nearest crisis management center in the study area. Figure 1 shows the first study area and the initial position of the relief workers in the study area. 


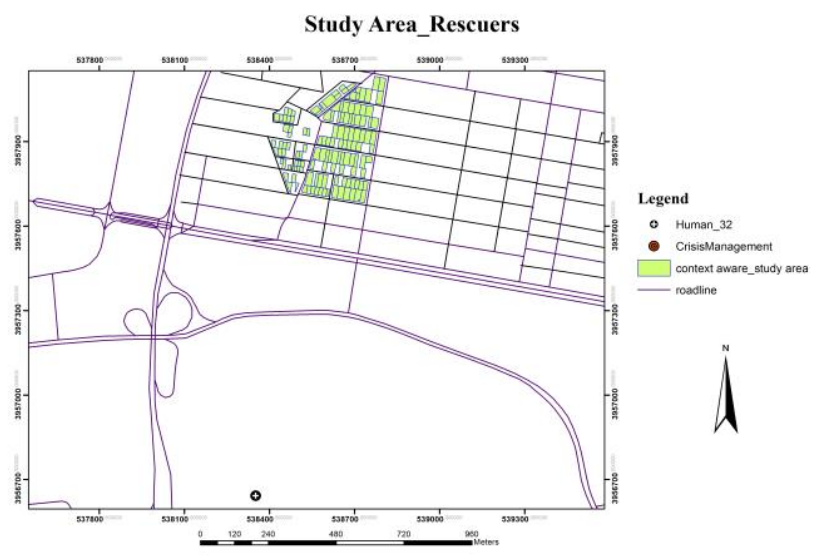

Figure 2: Study area and the first location of rescuers

The following figure shows the structure of the relief and rescue operations, based on the context information provided by the rescuers and the senses.

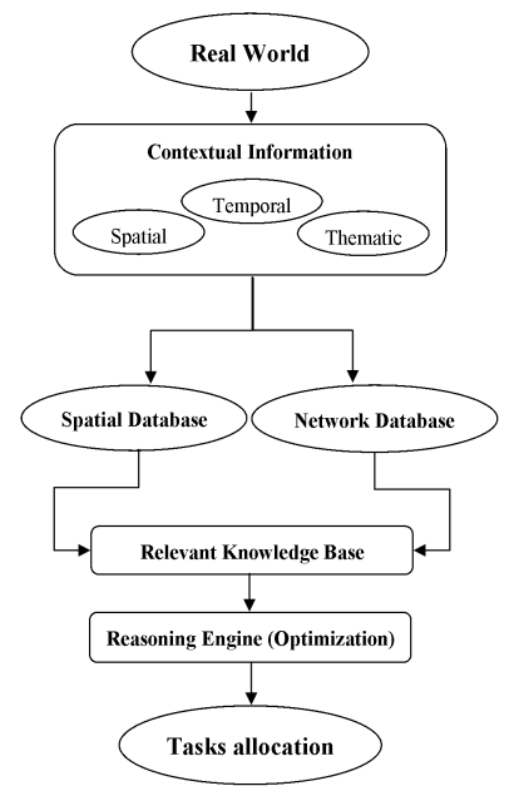

Figure 3: Context-aware relief \& rescue teams framework

In the following figure (Fig. 4), the pseudo-code implementation of the proposed context-aware algorithm is written to optimize post-earthquake relief and rescue operations.

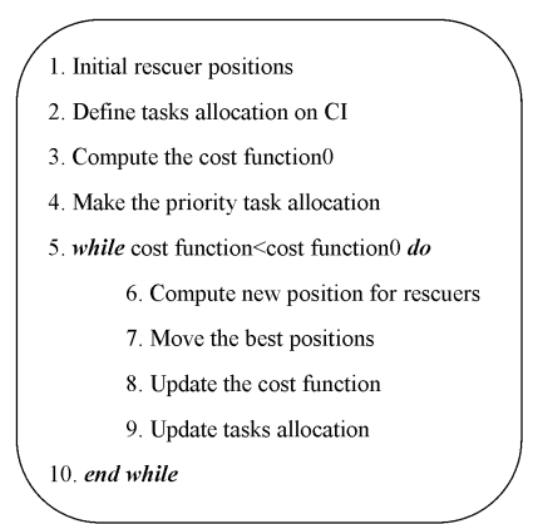

Figure 4: The pseudo-code of the context-aware algorithm

The following shows the descriptive information of relief workers in a hypothetical earthquake, in which 14 relief workers out of 32 relief workers, as well as the third, are shown their activities:

\begin{tabular}{|c|c|c|c|c|}
\hline UIDhuman & TypeHuman & Proficiency1 & Proficiency2 & Proficiency 3 \\
\hline 001 & Rescuer3 & Primary Helping & & \\
\hline 002 & Rescuer2 & Primary Helping & & \\
\hline 003 & Rescuer1 & & Primary Helping & \\
\hline 004 & Savior3 & Searching & Pointing & Secuting \\
\hline 005 & Savior2 & Searching & Pointing & Secuting \\
\hline 006 & Savior1 & Searching & Pointing & Secuting \\
\hline 007 & ISAR & Collapse Lifting & Secuting Pilot & $\begin{array}{l}\text { Air Update in the } \\
\text { Rubble }\end{array}$ \\
\hline 008 & Rescuer3 & Primary Helping & & \\
\hline 009 & Rescuer2 & Primary Helping & & \\
\hline 010 & Rescuer1 & r & Primary Helping & \\
\hline 011 & Savior3 & Searching & Pointing & Secuting \\
\hline 012 & Savior2 & Searching & Pointing & Secuting \\
\hline 013 & Savior1 & Searching & Pointing & Secuting \\
\hline 014 & ISAR & Collapse Lifting & Secuting Pilot & $\begin{array}{l}\text { Air Update in the } \\
\text { Rubble }\end{array}$ \\
\hline
\end{tabular}

Figure 5: Descriptive information of relief workers

Regarding the parameters stated in the method of implementation (i.e.; the descriptive information of the rescuers, the activities and initial damages of the earthquake and other Cis), the context aware PSO algorithm of this research, is evaluated and calculated using relations 1,2 and 3 for all the rescuers in comparison with all residential areas and ultimately, the optimal allocation of relief workers to the activities is obtained. An example of the optimal mode of relief and rescue teams is showing in the figure below.

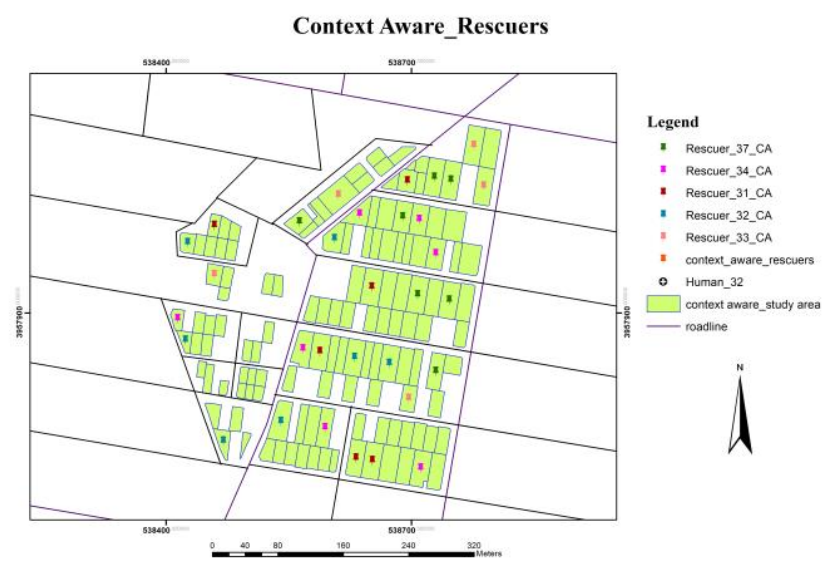

Figure 6: Context-aware Optimization of the Relief \& Rescue Team

In this implementation, data tables have entered the particle swarm algorithm, which has transformed from continuous to the discrete mode for the response space. According to the results obtained in the implementation of the algorithm with various CIs, its outputs were recorded and displayed. In order to control the accuracy of the results and prevent the rapid convergence of the algorithm, the coefficient $\mathrm{W}$ is equal to 1 , and the coefficients $C_{1}$ and $C_{2}$ are equal to 2 (collectively equal to 4 , which are typically each equal to 2 ), and the coefficient of reduction $\mathrm{W}$ will be considering as 0.05 in subsequent iterations.

In the study area of the image above, the "Rescuers 34" relate to relief workers assigned to Light Collapse Lifting activities; "Rescuers32", relief workers, and Pointing; 
"Rescuers31", rescuers assigned to Searching activities. As well as "Rescuers33" for rescue workers who are engaged in Securing Pilot and rescue workers "Rescuers 37", engaged in Primary Helping activities. The allocation of people is carried out according to the priority, and the residential areas that have more damage are in the priority of the relief effort.

In evaluating the efficiency of the proposed algorithm, the positive effect of the initial population selection method shown in the results obtained from the implementation of the proposed algorithm. Finally, a 2.4 fold improvement in the results obtained from the state that was not used by this algorithm. In the table below, the calculation of the cost function in the two modes of implementation of the proposed algorithm and its non-implementation is set, which represents the calculated cost of the allocation in the two situations for the entire operational team.

\begin{tabular}{|c|c|}
\hline Used model & $\begin{array}{c}\text { Cost calculated for the } \\
\text { entire operational team }\end{array}$ \\
\hline \hline $\begin{array}{c}\text { Without using the proposed } \\
\text { algorithm }\end{array}$ & 0.60 \\
\hline Using the proposed algorithm & 0.25 \\
\hline
\end{tabular}

Table 1: Comparison of the results of the proposed algorithm and its validation

\section{CONCLUSION}

Particle Swarm Algorithm is an algorithm based on collective intelligence, in which individual and group experiences are for optimal positioning. Therefore, due to the facts that the problem is considered to be grouped, the context awareness of the subject of this research, the effectiveness of each person's activity on the other people's activities and the group and the category of operations, as well as the structure of the particle swarm algorithm, which allows for more repetition in less time, the proposed algorithm of this study is identified as an appropriate solution to the post-earthquake relief and rescue problem.

The structure of the particle swarm algorithm is continuous; because of the discrete structure of the present, it is implemented discretely by applying changes to the structure of this algorithm. As previously stated, the context of individuals, their specializations, the activities, and the damaged sites have the same priorities as those that were implementing in the algorithm. Using the proposed algorithm of this research and applying the changes expressed in it, in order to optimize and implement the scientific and practical structure of rescue and rescue operation activities and teams, is a novel and effective way to improve the quality of relief and rescue after it will be an earthquake. Finally, as shown in Table 1 in the findings, the proposed algorithm implementation in this study improved the $2.4 \%$ of the results from the allocation of relief workers to a state that was not used by the proposed algorithm of this study. For future researches, the optimization methods such as simulated annealing, ant colony, genetics are considered.

\section{REFERENCES}

Akbari, M., Rashidi, H. (2016), “A Multi-Objectives Scheduling Algorithm Based on Cuckoo Optimization for Task Allocation Problem at Compile Time in
Heterogeneous Systems", Expert Systems with Applications, Volume 60, PP. 234-248.

Argany, M., Mostafavi, M. A. (2018), “Develop a GIS-based context-aware sensor network deployment algorithm to optimize sensor coverage in an urban area", International Archives of the Photogrammetry, Remote Sensing and Spatial Information Sciences ISPRS Archives.

Argany, M., Mostafavi, M. A., Gagné, Ch., 2015. "Context-Aware Local Optimization of Sensor Network Deployment"; Journal of Sensor and Actuator Networks, 160-188.

Aghakhani, M. Nasrabadi, T. Vafaeinejad, A.R., (2018), "Assessment of the effects of land use scenarios on watershed surface runoff using hydrological modelling", Applied Ecology and Environmental Research, Vol. 16, No. 3, p.p: 2369-2389 .

Baffo, I., Carotenuto, P., Rondine, S. (2017) "An orienteering-based approach to manage emergency situation", Transportation Research Procedia, Volume 22, 2017, Pages 297-304.

Bahrami, N., 2019. "Using Tabu Search Algorithm and Geospatial Information System for Managing of the Relief and Rescue Teams"; Journal of Geomatics Science and Technology (JGST), Volume 8, Issue 3, 179-188.

Bolouri, S., Vafaeinejad, A.R., Alesheikh, A.A., Aghamohammadi, H., (2018), "The Ordered Capacitated Multi-Objective Location-Allocation Problem for Fire Stations Using Spatial Optimization", ISPRS International Journal of Geo-Information, Vol. 7, No. 2, pp: 44-64.

Eizadi, A. (2011), "Review over the basis of disaster management", Relief \& Rescue organization, Tehran.

Ghaderi, A. et al. (2012), "An Efficient Hybrid Particle Swarm Optimization Algorithm for Solving the Uncapacitated Continuous Location-Allocation Problem", Networks and Spatial Economics 12(3):119, September 2012.

Granmo, O., Radianti, J., Goodwin, M., Dugdale, J., Sarshar, P., Glimsdal, S., Gonzalez, J. J. (2013), “A Spatio-temporal Probabilistic Model of Hazard and Crowd Dynamics in Disasters for Evacuation Planning”, Applied Intelligence, January 2013. Volume 42. Issue 1. pp. 3-23.

Jena, R. K. (2015), "Multi Objective Task Scheduling in Cloud Environment Using Nested PSO Framework", Procedia Computer Science, Volume 57, 2015, Pages 1219-1227.

Lei X., Zhou, X. Z., Li, Q. M., Zhang, X. F. (2016), "Energy-efficient resource allocation for multiuser OFDMA system based on hybrid genetic simulated annealing", Soft Computing. PP. 1-8. 
Lin, J. T., Chiu, C. C. (2015) "A hybrid particle swarm optimization with local search for stochastic resource allocation problem", Journal of Intelligent Manufacturing, PP. 1-15.

Liu, Y. L. et al. (2012), "Rural land use spatial allocation in the semiarid loess hilly area in China: Using a Particle Swarm Optimization model equipped with multi-objective optimization techniques", Science China Earth Sciences, July 2012, Volume 55, Issue 7, pp. 1166-1177.

Liua, X., Lia, X., Shib, X., Huanga, K., Liua, Y. (2012), “ A multi-type ant colony optimization (MACO) method for optimal land use allocation in large areas", International Journal of Geographical Information Science, Volume 26, 2012 - Issue 7, Pages 1325-1343, Taylor and Francis Online.

Mahdjoub, J., Rousseaux F., Soulier E. (2014), "Towards Better Coordination of Rescue Teams in Crisis Situations: A Promising ACO Algorithm", Information Systems for Crisis Response and Management in Mediterranean Countries, Volume 196 of the series Lecture Notes in Business Information Processing PP. 135-142.

"Mountaineer Area Rescue Group. Probability of Detection”. Appalachian Search and Rescue Conference.

Mousanejad, A., Vafaeinejad, A., Eslami, K. (2018), "Using geographic information system and simulated annealing for optimizing the railway design", Lecture Notes in Computer Science (including subseries Lecture Notes in Artificial Intelligence and Lecture Notes in Bioinformatics).

Nedjah, N., Mathias, R., Mendonça, L., Mourelle, M. (2015), "PSO-based Distributed Algorithm for Dynamic Task Allocation in a Robotic Swarm", Procedia Computer Science, Volume 51, 2015, Pages 326-335.

Neysani Samany, N., Delavar, M.R., Chrisman, N., Malek, M.R. (2014), "FIA5: A customized Fuzzy Interval Algebra for modeling spatial relevancy in urban context-aware systems", Engineering Applications of Artificial Intelligence, Volume 33, August 2014, Pages 116-126.

Neysani Samany, N., Delavar, M.R., Chrisman, N., Malek, M.R. (2013), "Spatial relevancy algorithm for contextaware systems (SRACS) in urban traffic networks using dynamic range neighbor query and directed interval algebra", Journal of Ambient Intelligence and Smart Environments, vol. 5, no. 6, pp. 605-619.

Oh, G., Kim, Y., Ahn, J., Choi, H. L. (2016), "PSO-based Optimal Task Allocation for Cooperative Timing Missions", IFAC-PapersOnLine, Volume 49, Issue 17, 2016, Pages 314-319.

Radianti, J, Granmo, O. C., Sarshar, P., Goodwin, M., Dugdale, J., Gonzalez, J. J. (2015), "A spatio-temporal probabilistic model of hazard- and crowd dynamics for evacuation planning in disasters", Applied Intelligence, January 2015, Volume 42, Issue 1, pp. 3-23.
Rasekh, A., Vafaeinezhad, A. R. (2012), "Developing a GIS Based Decision Support System for Resource Allocation in Earthquake Search and Rescue Operation", Computational Science and Its Applications - ICCSA 2012, Volume 7334 of the series Lecture Notes in Computer Science PP. 275-285.

Saeedian, B. et al. (2016), "Comparison of the efficiency of genetic and mass fragmentation algorithms for optimal allocation of water to agricultural land under water constraints". Journal of Information Technology Engineering, Vol. 3, No. 4, pp. $19-42$.

Sajadian, B. M., Vafaeinejad, A. R., Alesheikh, A. A., 2017. "Designing a context-aware recommender system in terms of air pollution"; Scientific - Research Quarterly of Geographical Data (SEPEHR), Vo. 26, No. 101, 61-71, Spring.

Shin, Y., Kim, S., Moon, I., (2019), "Integrated optimal scheduling of repair crew and relief vehicle after disaster", Computers \& Operations Research, Volume 105, May 2019, Pages 237-247.

Tang Jian, Zhu Kejun, Guo Haixiang, Gong Chengzhu, Liao Can, Zhang Shuwen, (2018), " Using auctionbased task allocation scheme for simulation optimization of search and rescue in disaster relief", Simulation Modelling Practice and Theory, Volume 82, March 2018, Pages 132-146.

Wang, H., Xu, R., Zijie, X., Zhou, X., Wang, O., Duan, Q., Bu, X. (2018), "Research on the Optimized Dispatch and Transportation Scheme for Emergency Logistics", Procedia Computer Science, Volume 129, 2018, Pages 208-214.

Wei Hong Lim, Nor Ashidi Mat Isa, (2015), "Particle swarm optimization with dual-level task allocation", Engineering Applications of Artificial Intelligence, Volume 38, February 2015, Pages 88-110.

Vafaeinezhad, A.R., (2018), "Design and implementation of a dynamic GIS with emphasis on navigation purpose in urban area", Lecture Notes in Computer Science (including subseries Lecture Notes in Artificial Intelligence and Lecture Notes in Bioinformatics).

Vafaeinezhad, A.R., (2017), "Dynamic Guidance of an Autonomous Vehicle with Spatio-Temporal GIS", Lecture Notes in Computer Science, Vol. 10407, Pp: 502-511.

Vafaeinezhad, A. R. Alesheikh, A. A. Nouri, J., (2010), "Developing a spatio-temporal model of risk management for earthquake life detection rescue team. International Journal of Environmental Science \& Technology". March 2010, Volume 7, Issue 2, pp. 243 250 . 
Vafaeinezhad, A.R., Alesheikh, A.A., Roshannejad, A.A., Shad, R., 2009. "A new approach for modeling spatio-temporal events in an earthquake rescue scenario", Journal of Applied Sciences, 9: 513-520.

Vafaeinezhad, A.R. Alesheikh, A.A. Hamrah, M. Reza Nourjou, R. Shad, R. (2009), "Using GIS to Develop an Efficient Spatio-temporal Task Allocation Algorithm to Human Groups in an Entirely Dynamic Environment Case Study: Earthquake Rescue Teams". Computational Science and Its Applications - ICCSA 2009. Volume 5592 of the series Lecture Notes in Computer Science. pp. 66-78.

Vahidnia, M.H., Vafaeinejad, A.R., Shafiei, M., (2019), "Heuristic game-theoretic equilibrium establishment with application to task distribution among agents in spatial networks", Journal of Spatial Science, Vol. 64, No.1, pp:131-152.

Valadbeygi, B., Pour Heydari, Gh. (2011), "Response and Recovery in crises", Iranian Crescent Institute of Applied Science and Technology \& Baqiyatallah University of Medical Sciences, Tehran.

Zhang, H., Xie, J., Ge, J., Lu, W., Zong, B. (2018), “An Entropy-based PSO for DAR task scheduling problem", Applied Soft Computing, Volume 73, December 2018, Pages 862-873. 